\title{
Indoor calibration method for UV index meters with a solar simulator and a reference spectroradiometer
}

\author{
Helmi Zaini ${ }^{1,2,3}$, Jae-Keun Yoo ${ }^{1,2}$, Seongchong Park ${ }^{1}$, and Dong-Hoon Lee ${ }^{1,2, \star}$ \\ 1 Korea Research Institute of Standards and Science, 305-340 Daejeon, Korea \\ 2 Korea University of Science and Technology, 305-340 Daejeon, Korea \\ 3 Puslit Metrologi LIPI, 15314 Tangerang Selatan, Indonesia
}

Received: 12 November 2015 / Accepted: 23 December 2015

\begin{abstract}
We present a calibration method of UV index meters under a solar simulator based on a spectroradiometer as reference. The reference spectroradiometer is a double-grating-based type that can measure the erythemal effective irradiance and the global UV index in a wavelength range from $250 \mathrm{~nm}$ to $450 \mathrm{~nm}$. After calibration of its wavelength scale and spectral irradiance scale by using the KRISS standard lamp, we applied the spectroradiometer to measure the reference value for calibrating broadband UV index meters under a solar simulator. The uncertainty of the spectroradiometer for measuring the reference value for the global UV index is evaluated to be $5 \%$ as a relative expanded uncertainty $(k=2)$.
\end{abstract}

Keywords: Global UV index, spectroradiometer, erythemal effective irradiance

\section{Introduction}

Considering the significant effects of the ultraviolet (UV) radiation on living organisms [1], quantitative monitoring of the solar UV radiation is a global issue that is closely related to the environment and the health of humans [2]. The global UV index is a quantitative index for reporting the level of harmful ambient solar UV radiation to the general public, which is standardized and adopted by the world health organization (WHO), the world meteorological organization (WMO), and the united nations environment programme (UNEP), and the international nonionizing radiation protection commission (ICNIRP) [3,4]. It is designed to represent the exposure of the skin to UV radiation in a single number by applying the erythema action spectrum [5].

Two types of instruments are used in practice for measuring the global UV index: a spectroradiometer [6] and a broadband filter radiometer [7]. The spectroradiometer type measures the spectral irradiance of the solar UV radiation by scanning a wavelength band, and provides the data of the erythemal effective irradiance required for determining the global UV index. The broadband filter radiometer type, on the other hand, directly indicates a single number that is proportional to the global UV index, when its responsivity is closely matched to the erythema action spectrum. Due to their fast response, simple operation, and small size, the broadband filter radiometer-type instruments, which we simply refer to as UV index meters, are widely used for monitoring solar UV radiation on a continuous basis in the field. However, in order to

${ }^{\star}$ Correspondence: dh.lee@kriss.re.kr achieve high-quality measurement and monitoring of solar UV radiation, a reference spectroradiometer carrying the absolute scale of spectral irradiance is required for calibrating the UV index meters used in the field $[8,9]$.

In this work, we present a calibration method of broadband UV index meters by comparison with a reference spectroradiometer under a solar simulator, and the progress of its experimental realization at the Korea research institute of standards and science (KRISS) The reference spectroradiometer measures the UV radiation and the global UV index of the solar simulator by recording the spectral irradiance in a wavelength range from $250 \mathrm{~nm}$ to $450 \mathrm{~nm}$. The setup and calibration of the reference spectroradiometer is performed similarly to the traveling reference spectroradiometer of the World Radiation Center (WRC) at Davos [10,11]. The focus of our work at the current stage is to test the feasibility of the indoor calibration of broadband UV index meters under a solar simulator. This method can be an alternative approach to the quality assurance of the UV index meters based on the outdoor calibration in Europe [8,9].

\section{Reference spectroradiometer}

Figure 1 schematically shows the configuration of the UV reference spectroradiometer developed at KRISS together with its photograph. The main part is a scanningtype double-grating monochromator (Bentham, model DMc150) consisting of two symmetrical Czerny-Turner monochromator arranged for additive dispersion mode. Each monochromator unit has a 1200 line/mm grating, a focal length of $150 \mathrm{~mm}$, and an aperture ratio of $f / 4$. The widths of both the input and output slits are adjusted 


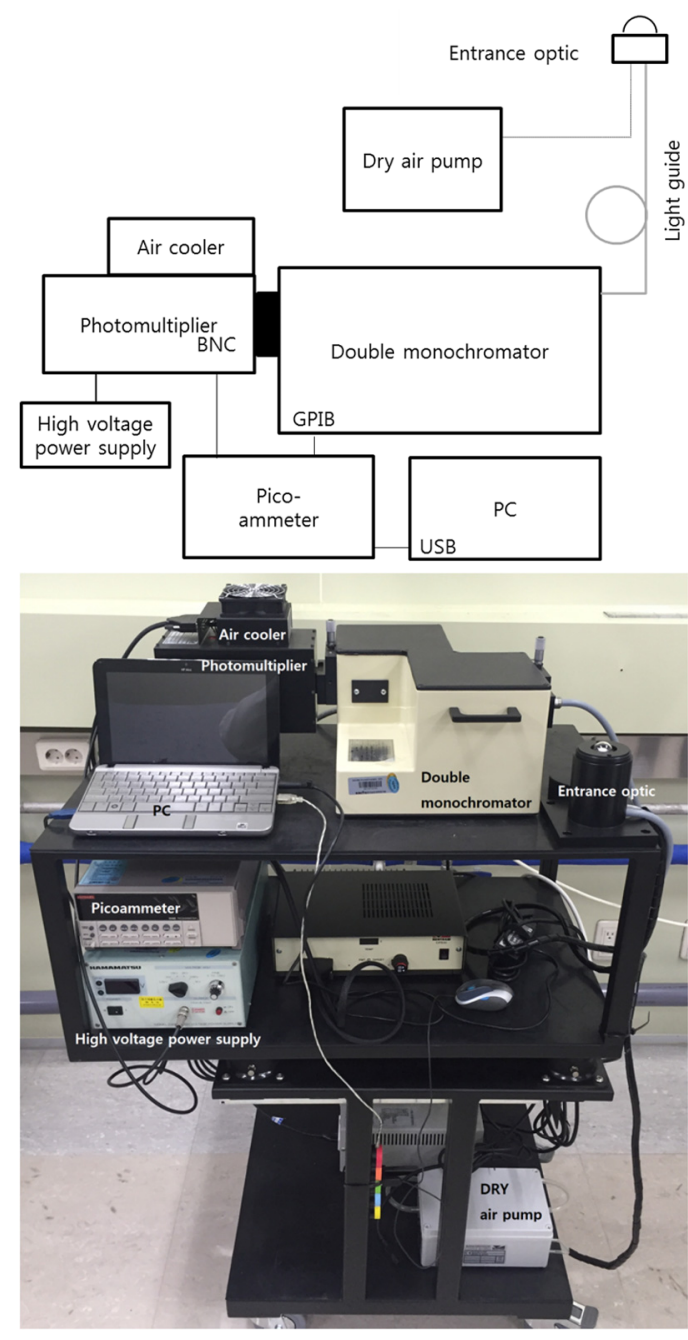

Fig. 1. Schematic configuration diagram and a photograph of the UV reference spectroradiometer at KRISS (see text for details).

to $0.5 \mathrm{~mm}$ so that the resulting spectral bandwidth of the double monochromator is less than $3 \mathrm{~nm}$ in full width at half-maximum (FWHM) in a wavelength range from $250 \mathrm{~nm}$ to $400 \mathrm{~nm}$ which is relevant for the global UV index measurement In this wavelength range, no order sorting filter is used since no harmonic signal can appear.

The incident light to the monochromator is received by the entrance optic consisting of a diffuser and a quartz dome window and delivered to the monochromator via a 2-m long flexible light guide (Bentham, model DIFF D6 ENV). The dome has a function to prevent the contamination and to anticipate the condensation of the diffuser by circulating dry air. The angular response of the entrance optic as an irradiance-measuring detector is close to a cosine response, which is certified by the manufacturer with a value of integrated cosine error ( $f_{2}$ parameter $)$ of 0.997 [12].

As the detector of the spectroradiometer, a thermoelectrically cooled end-window-type bi-alkali photomultiplier tube (PMT, Bentham, model DH-50(Bi)-Te) is used at the output slit of the monochromator. It is operated by supplying a constant high voltage of $700 \mathrm{~V}$, and the specified sensitive wavelength range of the PMT is from $200 \mathrm{~nm}$ to $600 \mathrm{~nm}$. The photocurrent from the PMT is measured by using a picoammeter (Keithley, model 6485). The spectroradiometer as a system is controlled by a computer via IEEE 488.2-GIPB and USB interfaces. We also developed measurement software that realizes automated calibration and measurement procedures of the spectroradiometer as well as recording and evaluation of the measured data.

As the PMT detector is used as a part of the spectroradiometer, the nonlinearity and stability of its response are the important characteristics affecting the accuracy of the instrument. The temperature stability of the PMT detector is specified to be below $1 \% /{ }^{\circ} \mathrm{C}$ at beyond $800 \mathrm{~nm}$ by the manufacturer, which implies that its effect is very small for the use in the UV range below $450 \mathrm{~nm}$ in the stable laboratory environment. However, the nonlinearity of the response can cause a measurement error and uncertainty, which will be discussed in Section 3.2.

Before the spectroradiometer is used for measurement, the basic calibration procedures should be performed for its wavelength scale and spectral irradiance scale. These are described in the following sub-sections.

\subsection{Wavelength scale calibration}

The wavelength scale of the UV reference spectrometer is calibrated with respect to the atomic spectral lines by using an $\mathrm{Hg}-\mathrm{Ne}$ pencil-type calibration lamp (Newport, model 6034). For calibration, the spectral distribution of the calibration lamp placed close to the entrance optic is measured by scanning from $250 \mathrm{~nm}$ to $450 \mathrm{~nm}$ with steps of $0.1 \mathrm{~nm}$. The analytic relationship between the grating angle of the monochromator and the atomic reference data of selected spectral lines is obtained by fitting a third-order polynomial function.

Figure 2 shows the result of the wavelength scale calibration, which is the spectral distribution of the $\mathrm{Hg}-\mathrm{Ne}$ pencil-type calibration lamp measured with the UV reference spectroradiometer. The measured PMT photocurrent in a logarithmic scale is plotted against the calibrated wavelength scale. The values at the cross symbols are the reference peak wavelengths of the atomic emission line of Mercury (Hg-I), which are selected and used for calibration [13]. From a series of calibration measurements, we verified that the differences between the measured and the reference peak values of the atomic spectral lines lie within an interval of $\pm 0.2 \mathrm{~nm}$. From analysis of the shape of each isolated spectral line, we also confirmed a nearly triangular slit function with a FWHM spectral bandwidth varying in a range from $2.0 \mathrm{~nm}$ to $2.5 \mathrm{~nm}$ depending on wavelength.

\subsection{Spectral irradiance scale calibration}

Calibration of the spectral irradiance scale means that we determine the responsivity of the PMT photocurrent reading against spectral irradiance at the entrance optic in 


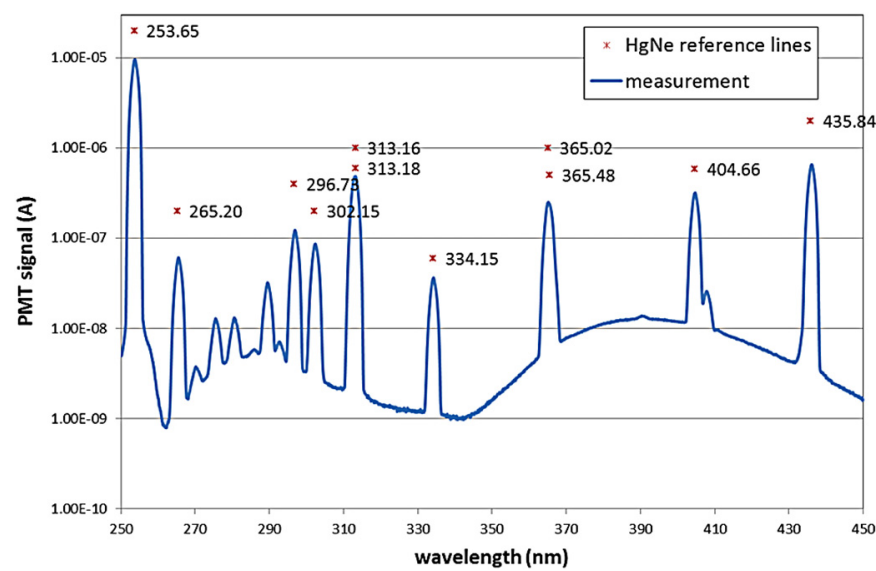

Fig. 2. Spectral distribution of an Hg-Ne pencil-type discharge lamp measured with the UV spectroradiometer after the wavelength scale calibration. The cross symbols indicate the atomic reference values used for calibration.

the unit of $\mathrm{A} /\left(\mathrm{W} \mathrm{m}^{-2} \mathrm{~nm}^{-1}\right)$. For this purpose, the UV spectroradiometer is mounted on a photometric bench to measure spectral irradiance of a reference standard lamp that is a $1 \mathrm{~kW}$ FEL-type quartz-tungsten-halogen (QTH) lamp calibrated at KRISS [14]. The spectroradiometer was calibrated at a distance of $50 \mathrm{~cm}$ between the reference plane of the standard lamp and the tip of the diffuser in the entrance optic. The calibration measurement was carried by scanning from $250 \mathrm{~nm}$ to $450 \mathrm{~nm}$ with steps of $1 \mathrm{~nm}$.

Figure 3 shows the results of the spectral irradiance scale calibration: Figure 3a is the PMT photocurrent signal as a function of wavelength measured for the spectral irradiance standard lamp, Figure $3 \mathrm{~b}$ the reference data of the standard lamp, and Figure 3c the spectral irradiance responsivity of the UV spectroradiometer calculated by dividing the data of Figure 3a by those of Figure 3b. The blue lines and symbols in Figure 3 are the data assigned to the left $y$-axis, while the red dashed lines and symbols depict the expanded relative uncertainties assigned to the right $y$-axis. All the uncertainties values plotted in Figure 3 are evaluated for a level of confidence of approximately $95 \%$ with a coverage factor $k=2$ [15]. In Figure 3b, the certified reference data of the standard lamp is shown as symbols, while the blue line is the cubic-spline interpolated data for an interval of $1 \mathrm{~nm}$. The data in Figure 3c are obtained by dividing the data in Figure 3a by those in Figure $3 \mathrm{~b}$ at each wavelength. The detailed procedure and uncertainty analysis of spectral irradiance scale calibration of a spectroradiometer can be found, for example, in reference [16].

\section{Solar simulator measurement}

In this section, we describe the measurement result of the global UV index of a solar simulator by using the UV reference spectroradiometer, and its uncertainty analysis. We note that this is the step establishing a reference scale
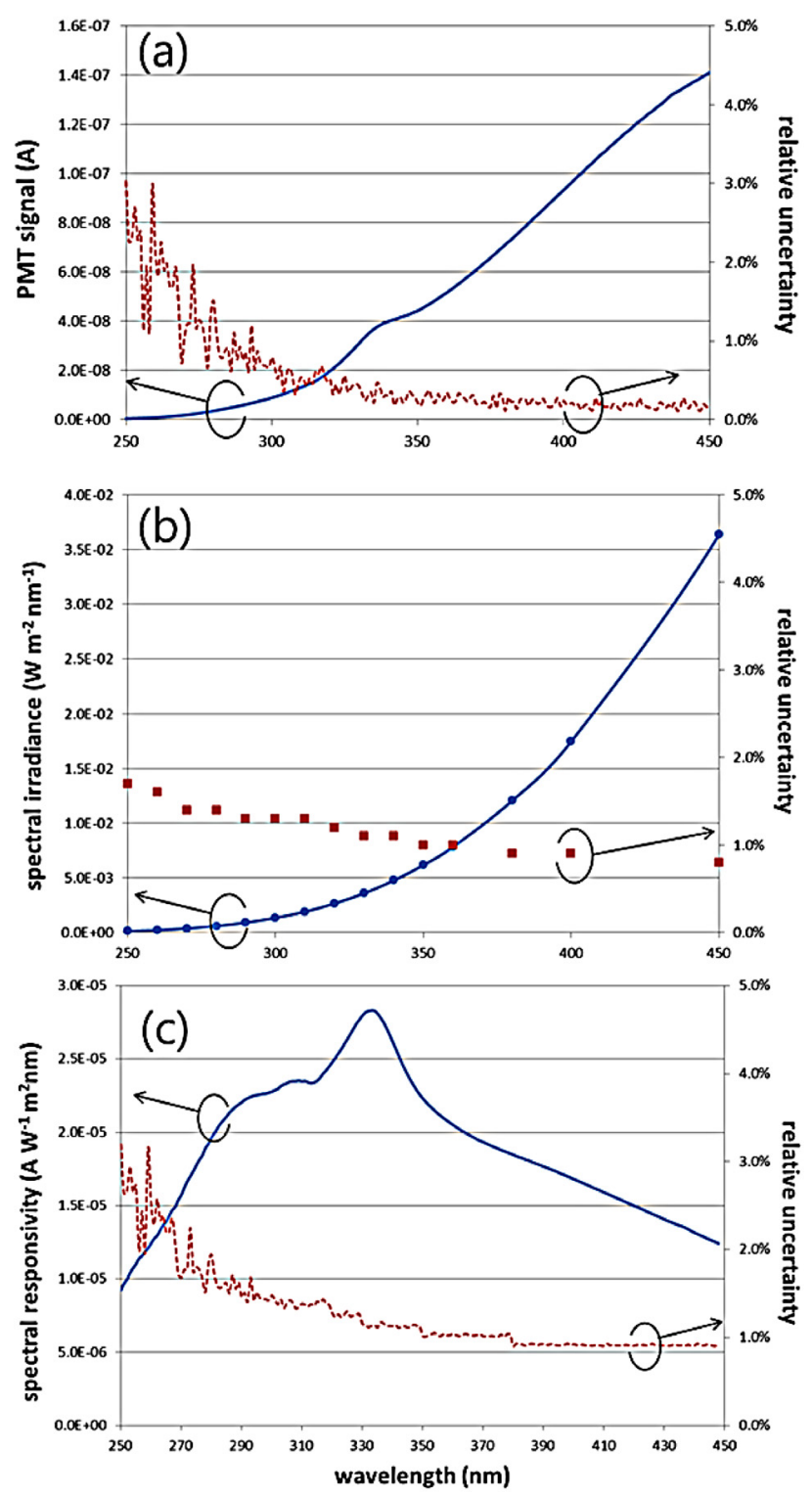

Fig. 3. Results of the spectral irradiance scale calibration of the UV reference spectroradiometer; (a) PMT photocurrent reading (blue line) with its repeatability uncertainties (red dashed line) for measurement of the spectral irradiance standard lamp, (b) the certified reference data of the standard lamp (blue dot symbols) with its uncertainties (red square symbols) and the interpolated data (blue line), (c) spectral irradiance responsivity of the spectroradiometer (blue line) with its uncertainties (red dashed line) calculated from (a) and (b).

for the proposed indoor calibration method of broadband UV index meters.

The global UV index $I_{U V}$ is defined as follow [3]:

$$
I_{U V}=k_{e r} \int_{250}^{400} E(\lambda) \cdot s_{e r}(\lambda) d \lambda .
$$

Here, $E(\lambda)$ denotes the spectral irradiance of the solar radiation in the unit of $\mathrm{W} /\left(\mathrm{m}^{2} \mathrm{~nm}\right)$ at wavelength of $\lambda$, and $k_{e r}$ a constant equal to $40 \mathrm{~m}^{2} / \mathrm{W}$ converting 
the erythemal weighted irradiance value to a common index number typically in a range from 0 to 10 . The function $s_{e r}(\lambda)$ in equation (1) is the erythema action spectrum given as $[4,5]$ :

$$
s_{e r}(\lambda)=\left\{\begin{array}{lcc}
1 & \text { for } & 250 \mathrm{~nm}<\lambda \leqslant 298 \mathrm{~nm} \\
10^{0.094(298-\lambda)} & \text { for } & 298 \mathrm{~nm}<\lambda \leqslant 328 \mathrm{~nm} \\
10^{0.015(139-\lambda)} & \text { for } & 328 \mathrm{~nm}<\lambda \leqslant 400 \mathrm{~nm} \\
0 & \text { for } & \lambda>400 \mathrm{~nm} .
\end{array}\right.
$$

Note that the integral quantity of equation (1) without the conversion constant $k$ is often referred to the erythemal effective irradiance $E_{e r}[3]$.

From equation (1), we see that the global UV index is determined readily from spectral irradiance measurement by using a UV spectroradiometer. However, we must be aware that the global UV index is defined only for the solar UV radiation reaching a horizontal outdoor surface from the total observed hemisphere. If one measures spectral irradiance $E(\lambda)$ under an indoor artificial light source such as a solar simulator, which mostly provides radiation in a beam, and calculates the quantity $I_{U V}$ according to equation (1), we cannot call it the global UV index of the solar simulator. Instead, we define in this work a new term called "the source UV index" with a symbol $I_{U V}^{*}$ as:

$$
I_{U V}^{*}=k_{e r} \int_{250}^{400} E^{*}(\lambda) \cdot s_{e r}(\lambda) d \lambda
$$

with $E^{*}(\lambda)$ indicating the spectral irradiance measured at a specified light source under a specific condition. The most important differences of the conditions between $E(\lambda)$ of the solar radiation and $E^{*}(\lambda)$ of the solar simulator are the spectral and spatial distribution of the incident radiation. The effects of these differences on the calibration of UV index meters will be discussed more in Section 4 . In this section, we consider only the determination of the source UV index of a solar simulator by measuring the spectral irradiance $E^{*}(\lambda)$ under a specific condition by using the UV reference spectroradiometer.

\subsection{Measurement result}

For experimental purpose, we used a small-area solar simulator based on a 100-W Xe arc lamp (Newport, model 94011 A of LCS-100 series) with a beam size of $38 \mathrm{~mm} \times 38 \mathrm{~mm}$. With a proper filter installed, the simulator provides a spectral match to the AM1.5G solar spectrum fulfilling the requirement for class A of the IEC classification [17]. The entrance optic of the spectroradiometer is horizontally placed at a distance of approximately $20 \mathrm{~cm}$ from the solar simulator output optics so that the output beam is normally incident to the diffuser of the entrance optic. The collimation angle of the beam is specified to be smaller than $6^{\circ}$ as a half angle according to the manufacturer. The irradiance level at the measurement plane was approximately $100 \mathrm{~mW} / \mathrm{cm}^{2}$ (=1 sun) at its maximum and could be adjusted with an iris aperture

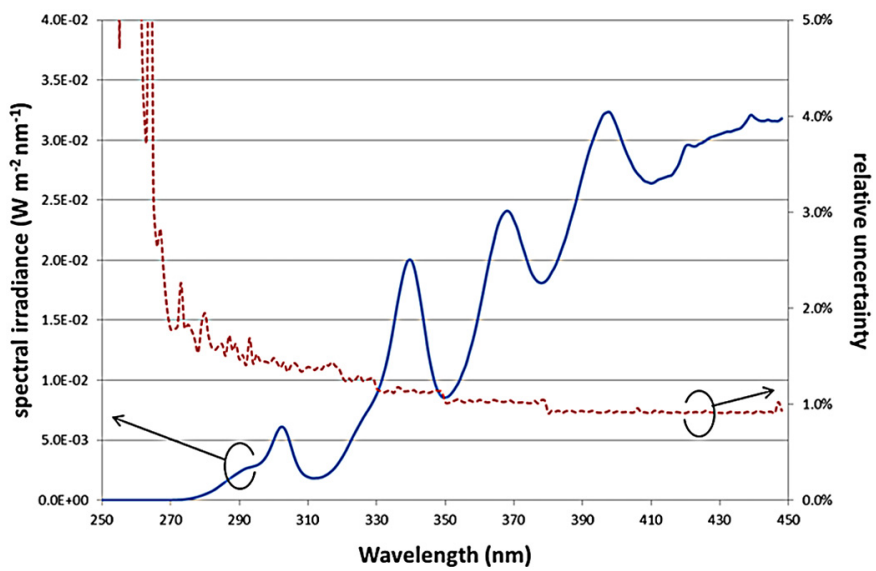

Fig. 4. Spectral irradiance of the solar simulator measured by using the UV reference spectroradiometer; the blue line shows the measured data in the left $y$-axis scale, while the red dashed line shows their expanded relative uncertainties in the right $y$-axis scale.

inside the solar simulator without changing the spectral distribution.

Figure 4 shows the measured spectral irradiance of the solar simulator (blue line) with its relative expanded uncertainties (red dashed line, $k=2$ ). Similar to Figure 3, the scales of the measurement data and the uncertainties are assigned to the left and right $y$-axis, respectively. For this measurement, the irradiance level of the solar simulator is adjusted so that the photocurrent of the PMT detector of the spectroradiometer is in the same range as the one used for the calibration measurement of Figure $3 \mathrm{a}$. The spectral irradiance for wavelengths below $270 \mathrm{~nm}$ is very low, which causes a large increase of the relative uncertainties up to $30 \%$ due to the extremely low signalto-noise ratio. The detailed uncertainty analysis will be described in the following sub-section. According to equation (2), we determine the source UV index $I_{U V}^{*}$ to be 2.40 from the measurement result of $E^{*}(\lambda)$ in Figure 4 for the solar simulator under the condition of normal incidence of a nearly collimated beam.

\subsection{Uncertainty analysis}

In the following, we discuss the dominant uncertainty components affecting the final result of the source UV index. Evaluation and expression of uncertainty is performed in accordance with the ISO guide [15].

\section{Uncertainty of spectral irradiance measurement}

The uncertainties of the spectral irradiance data measured with the spectroradiometer, which are shown in Figure 4, propagate to the uncertainty of the source UV index according to equation (2). Note that the uncertainties of spectral irradiance already include both the calibration uncertainties of the spectroradiometer (Fig. 3) and the uncertainties of reading repeatability in measurement. 
Since we operated the UV reference spectroradiometer with a scan step of $1 \mathrm{~nm}$ both for calibration and measurement, the integral equation (2) can be re-written to a summation with $\lambda$ as an integer index running from $250 \mathrm{~nm}$ to $400 \mathrm{~nm}$ :

$$
I_{U V}^{*}=k_{e r} \sum_{\lambda}\left[E_{\lambda}^{*} \cdot s_{e r, \lambda}\right]
$$

Assuming that the measured spectral irradiance data are fully correlated with respect to wavelength, we obtain a simple propagation equation for the standard uncertainty of the source UV index $u\left(I_{U V}^{*}\right)$ as:

$$
u\left(I_{U V}^{*}\right)=\sum_{\lambda}\left[c_{\lambda} \cdot u\left(E_{\lambda}^{*}\right)\right]
$$

with the absolute standard uncertainties of spectral irradiance $u\left(E_{\lambda}^{*}\right)$ and the sensitivity coefficients $c_{\lambda}$ given by:

$$
\left.c_{\lambda} \equiv \frac{\partial I_{U V}^{*}}{\partial E^{*}}\right|_{\lambda}=\left.k_{e r} \cdot s_{e r}\right|_{\lambda}
$$

For the result shown in Figure 4, the standard uncertainty of $I_{U V}^{*}$ propagated from the spectral irradiance measurement is evaluated to be 0.018 according to equations (4) and (5) with the normal probability distribution. We note that the assumption of the spectrally correlated data for equation (4) is justified from the strong spectral correlation of the spectral irradiance standard lamp [16].

\section{Uncertainty of wavelength scale}

The wavelength scale of the spectroradiometer is calibrated with the maximum error of $\pm 0.2 \mathrm{~nm}$ as described in Section 2.1. In order to estimate the maximum deviation of the source UV index due to the wavelength scale error, we intentionally introduced an offset error of $\pm 0.2 \mathrm{~nm}$ to the spectral irradiance data in equation (3) and calculated the resulting change of $I_{U V}^{*}$. The corresponding error of $I_{U V}^{*}$ was determined to be +0.03 and -0.04 for an error of $-0.2 \mathrm{~nm}$ and $+0.2 \mathrm{~nm}$, respectively, resulting in a standard uncertainty of $0.07 /(2 \sqrt{3})=0.02$ for the source UV index with the rectangular probability distribution.

\section{Uncertainty of measurement position}

For irradiance measurement, the position of the detector reference plane is critical, especially in the direction of the beam propagation. This leads to an uncertainty propagated from the uncertainty of the vertical position of the entrance optic of the spectroradiometer. As we have no precise tool to align and check the position of the diffuser surface enclosed by a quartz dome window, we experimentally determined the sensitivity of the source UV index measurement with respect to the distance between the diffuser and the solar simulator output. Assuming that the maximum error of reproduced vertical positioning is $\pm 3 \mathrm{~mm}$, we measured the corresponding variation

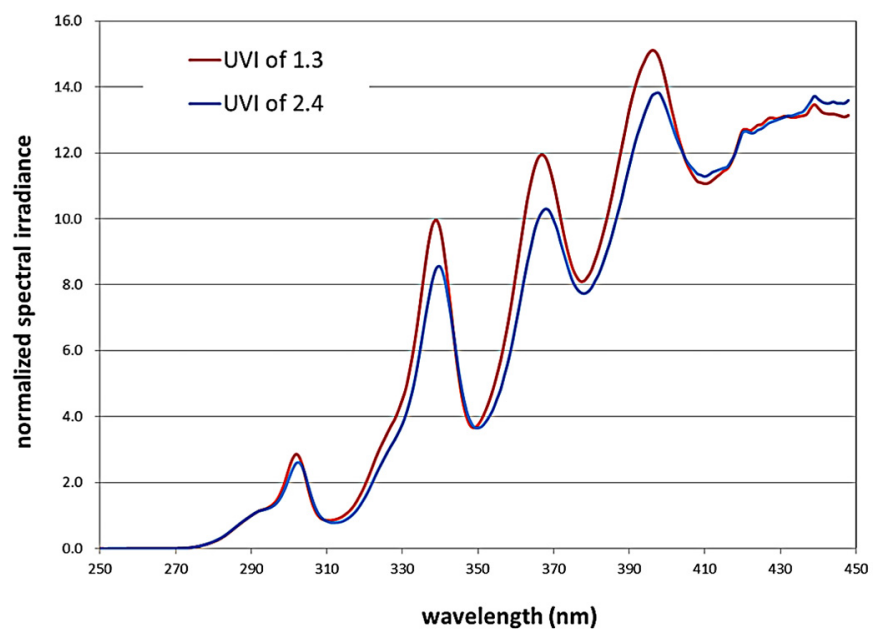

Fig. 5. Comparison of spectral distribution of the solar simulator at two different levels of irradiance measured with the UV reference spectroradiometer. The blue and red lines are measured for a source UV index level of 1.3 and 2.40, respectively, and plotted as the spectral irradiance normalized to the value at $290 \mathrm{~nm}$.

of $I_{U V}^{*}$ to be \pm 0.024 , resulting in a standard uncertainty of $0.024 /(\sqrt{3})=0.015$ for the source UV index with the rectangular probability distribution.

\section{Uncertainty of detector nonlinearity}

The UV reference spectroradiometer uses a PMT detector, whose response can be nonlinear as the photocurrent changes. This can be especially critical, when the spectral distribution of the solar simulator shown in Figure 4 has a completely different shape from the one of the calibration source shown in Figure 3a, although both measurements are performed in the same range of amplification. In order to evaluate this, we measured spectral irradiance of the solar simulator at two different irradiance levels, and checked the change of its relative shape. As the solar simulator used for the experiment can adjust the irradiance level by using a mechanical iris aperture without changing the lamp operation condition, we expect that the irradiance level does not influence the spectral distribution, i.e. the relative shape of spectral irradiance.

Figure 5 shows the results of spectral distribution measurement at a level with the source UV index of 1.3 (red line) and 2.4 (blue line). For this plot, the measured spectral irradiance is normalized to the value at $290 \mathrm{~nm}$, where the PMT photocurrent is lower than $50 \mathrm{nA}$ in both cases. We see in Figure 5 that the spectral distribution changes as the irradiance level changes, indicating that the response of the spectroradiometer is nonlinear. For a more accurate evaluation of the detector nonlinearity, the PMT of the spectroradiometer can be tested separately as described in reference [10]. In this work, however, we estimate the maximum uncertainty due to detector nonlinearity based on the measurement of Figure 5. Calculation of the source UV index from the measured normalized 
Table 1. Uncertainty budget for the source UV index measurement of a solar simulator with the UV reference spectroradiometer in the case where the measured value is 2.40 .

\begin{tabular}{ccc}
\hline $\begin{array}{c}\text { Uncertainty } \\
\text { component }\end{array}$ & $\begin{array}{c}\text { Standard } \\
\text { uncertainty }\end{array}$ & $\begin{array}{c}\text { Probability } \\
\text { distribution }\end{array}$ \\
\hline $\begin{array}{c}\text { Propagation from spectral } \\
\text { irradiance measurement } \\
\text { (including repeatability) }\end{array}$ & 0.004 & normal \\
Wavelength scale & 0.02 & rectangular \\
Measurement position & 0.015 & rectangular \\
Detector nonlinearity & 0.05 & rectangular \\
Combined standard uncertainty & 0.056 & normal \\
Expanded uncertainty & 0.12 & $k=2$ \\
\hline
\end{tabular}

spectral irradiance data in Figure 5 results in a maximum difference of the source UV index of $3.8 \%$ for the two irradiance levels, from which we determine the relative standard uncertainty of the source UV index due to detector nonlinearity to be $3.8 \% /(\sqrt{3})=2.2 \%$ with the rectangular probability distribution, corresponding to the absolute standard uncertainty of 0.05 for the source UV index of 2.40 .

\section{Uncertainty budget}

Table 1 summarizes the uncertainty components of the source UV index of the solar simulator described above, and shows its combined uncertainty. For the source UV index of 2.40, its expanded uncertainty is evaluated to be 0.12 with a confidence level of approximately $95 \%$ $(k=2)$, corresponding to a relative expanded uncertainty of $5 \%$.

\section{Indoor calibration of UV index meters}

Taking the source UV index of the solar simulator measured with the UV reference spectroradiometer as the reference value, we can now develop a calibration procedure for a broadband UV filter radiometer, simply referred to as UV index meter, in the indoor laboratory environment. As we briefly discussed in the previous section to equations (1) and (2), an instrument used for measuring the "global" UV index of the solar radiation cannot be used for measuring the "source" UV index of a solar simulator due to the difference of the spectral and spatial radiation distribution of two sources. Careful corrections are required to be able to compare the results under two different conditions.

The first step of the indoor calibration scheme is to compare the UV index meter as the device under test (DUT) with the UV reference spectroradiometer under a solar simulator. As a result of the first step, the responsivity of the DUT against the source UV index is obtained. The second step is to measure the angular and spectral response of the DUT by using a separate experimental setup in the laboratory. As a result, we obtain the angular response function (ARF) and the spectral response function

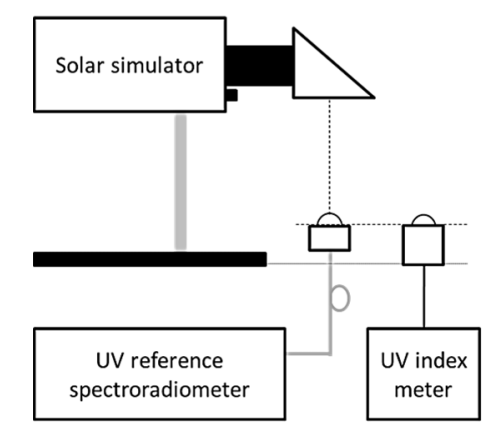

(1) Calibration against the source UV index

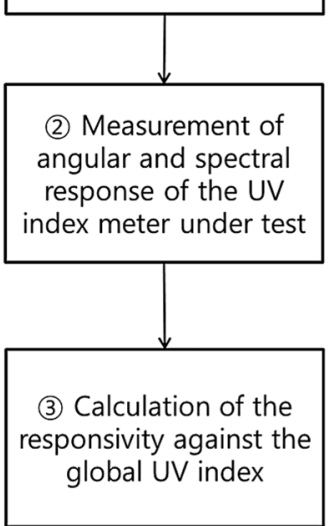

Fig. 6. Schematic setup and procedure of the proposed indoor calibration of UV index meters.

(SRF) of the DUT, which can be used to calculate the corresponding correction factors considering the standard solar spectrum, the standard erythemal action function, and the different solar zenith angles [8]. The third step is to apply these correction factors to calculate the response of the DUT to the global UV index from the measured responsivity to the source UV index.

Figure 6 shows the schematic setup and procedure of the indoor calibration for UV index meters proposed at KRISS. We used the solar simulator described in Section 3.1 for the indoor calibration. In this work, we are reporting the first step of the indoor calibration scheme in Figure 6. Nevertheless, by using a calibrated UV index meter that provides information on its ARF and SRF, we can experimentally test the feasibility of the calibration scheme with the setup for the first step. For this purpose, we selected a commercially available UV index meter (Kipp \& Zonen, model UVS-E-T), which provides the detailed information of calibration and is widely used in practice. The ARF of this type of UV index meters is reported to be very close to the cosine function for zenith angle of lower than $20^{\circ}$ [8] so that the cosine error can be neglected for the normal incident beam of the solar simulator with a collimation angle of less than $6^{\circ}$. The manufacturer provides the SRF data from $280 \mathrm{~nm}$ to $400 \mathrm{~nm}$ separated from the absolute responsivity against spectral irradiance at one wavelength $(292 \mathrm{~nm})$ so that we can calculate the spectral mismatch correction factor $f_{S}^{*}$ of the DUT against the measured spectral irradiance $E^{*}(\lambda)$ of the solar simulator according to the equation [8]:

$$
f_{S}^{*}=\frac{\int s_{e r}(\lambda) \cdot E^{*}(\lambda)}{\int S R F(\lambda) \cdot E^{*}(\lambda)} .
$$

The uncertainty of the spectral mismatch correction factor is dominantly propagated from the uncertainty of the $\mathrm{SRF}$, which is, however, not reported by the manufacturer.

Considering this spectral mismatch correction factor, the UV index meter measures the source UV index of the solar simulator, which can be directly compared with 
Table 2. Result of comparison of the source UV index between the reference spectroradiometer calibrated at KRISS and the broadband UV index meter calibrated by the manufacturer Kipp \& Zonen under a solar simulator. For spectral mismatch correction of the UV index meter, the spectral response function provided by the manufacturer is used.

\begin{tabular}{cccc}
\hline & $\begin{array}{c}\text { Source UV index } \\
\text { measured with } \\
\text { spectroradiometer with } \\
\text { expanded uncertainty } \\
\text { (traceable to KRISS) }\end{array}$ & $\begin{array}{c}\text { Source UV index } \\
\text { measured with } \\
\text { broadband UV } \\
\text { index meter }\end{array}$ & Difference \\
(traceable to manufacturer) & \\
\hline $\begin{array}{c}\text { Low irradiance } \\
\text { level }\end{array}$ & $1.29 \pm 0.07$ & 1.37 & $\begin{array}{c}-0.08 \\
(-6.2 \%)\end{array}$ \\
\hline $\begin{array}{c}\text { High irradiance } \\
\text { level }\end{array}$ & $2.40 \pm 0.12$ & 2.53 & $\begin{array}{c}-0.13 \\
(-5.4 \%)\end{array}$ \\
\hline
\end{tabular}

the results of the reference spectroradiometer. In experiment, we operated the solar simulator at the source UV in$\operatorname{dex} I_{U V}^{*}$ of 1.3 and 2.4 , which correspond to the source erythemal effective irradiance of $1.62 \mathrm{~W} / \mathrm{m}^{2}$ and $2.94 \mathrm{~W} / \mathrm{m}^{2}$, respectively. The temperature and the relative humidity of the laboratory environment are maintained within the ranges $(23 \pm 2){ }^{\circ} \mathrm{C}$ and $(45 \pm 15) \%$, respectively, during the measurement. Table 2 summarized the comparison results at two irradiance levels. The source UV index measured with the UV index meter is by $5.4 \%-6.2 \%$ higher than that measured with the reference spectroradiometer, which are slightly larger than the uncertainty of the spectroradiometer measurement. We note that the result of the validity test in Table 2 does not consider the uncertainty of the spectral mismatch correction because no information on the accuracy of the SRF of the DUT is available. A more accurate verification of the calibration can only be possible after the completion of the step 2 in Figure 6 .

\section{Summary}

We tested the feasibility of the calibration method of broadband UV index meters under a solar simulator by using a reference spectroradiometer. The reference spectroradiometer working in the UV range from $250 \mathrm{~nm}$ to $450 \mathrm{~nm}$ is calibrated against the spectral irradiance scale of KRISS and used to measure the UV radiation and the erythemal effective irradiance of a solar simulator in the laboratory. The "source" UV index, which we defined to distinguish the different spectral and spatial conditions between an indoor light source and the natural sun, is measured with a relative uncertainty of $5 \%(k=2)$ by using the reference spectroradiometer. The most dominant uncertainty component was the detector nonlinearity. We tested its validity by calibrating a UV index meter at two different irradiance levels under the solar simulator based on the data provided by the manufacturer.

Acknowledgements. This work was supported by the Korea Research Institute of Standards and Science under the project "Establishment of National Physical Measurement Standards and Improvements of Calibration/Measurement Capability", Grant 15011018.

\section{References}

1. P. Weihs, A.W. Schmalwieser, G. Schauberger, UV effect on living organism, Encyclopedia of sustainability science and technology, edited by R.A. Meyers (Springer, New York, 2012), pp. 11375-11427

2. CIE 209:2014, Rationalizing nomenclature for $U V$ doses and effects on humans, Joint publication of CIE and WMO; WMO/GAW Report No. 211 (International Commission on Illumination, Vienna, 2014)

3. CIE S 013/E:2003, International standard global solar UV index (International Commission on Illumination, Vienna, 2003)

4. ISO 17166:1999/CIE S 007/E-1998, Erythema reference action spectrum and standard erythema dose (International Organization for Standardization, Geneva, 1999)

5. A.F. McKinlay, B.L. Diffey, A reference action spectrum for ultraviolet induced erythema in human skin, CIE J. 6, 17-22 (1987)

6. G. Steckmeyer, A.F. Bais, G. Bernhard, M. Blumthaler, C.R. Booth, P. Disterhoft, P. Eriksen, R.L. McKenzie, M. Miyauchi, C. Roy, Instruments to measure solar ultraviolet radiation. Part 1. Spectral instruments, WMO/GAW Rep. 125 (World Meteorological Organization, 2001)

7. G. Steckmeyer, A.F. Bais, G. Bernhard, M. Blumthaler, C.R. Booth, R.L. Lantz, R.L. McKenzie, P. Disterhoft, A. Webb, Instruments to measure solar ultraviolet radiation. Part 2. Broadband instruments measuring erythemally weighted solar irradiance, WMO/GAW Rep. 164 (World Meteorological Organization, 2007)

8. G. Hülsen, J. Gröbner, Characterization and calibration of ultraviolet broadband radiometers measuring erythemally weighted irradiance, Appl. Opt. 46, 5877-5886 (2007)

9. J. Gröbner, M. Blumenthaler, S. Kazadzis, A. Bais, A. Webb, J. Schreder, G. Seckmeyer, D. Rembges, Quality assurance of spectral solar UV measurements: results from 25 UV monitoring sites in Europe, 2002 to 2004, Metrologia 43, S66-S71 (2006)

10. J. Gröbner, J. Schreder, S. Kazadzis, A.F. Bais, M. Blumthaler, P. Görts, R. Tax, T. Koskela, G. Seckmeyer, A.R. Webb, D. Rembges, Traveling reference spectroradiometer for routine quality assurance of spectral solar ultraviolet irradiance measurements, Appl. Opt. 44, $5321-5331(2005)$

11. J. Gröbner, P. Sperfeld, Direct traceability of the portable QASUME irradiance scale to the primary irradiance standard of the PTB, Metrologia 42, 134-139 (2005) 
12. ISO/CIE 19476:2011(E), Characterization of the performance of illuminance meters and luminance meters, Joint ISO/ICE international standard (International Commission on Illumination, Vienna, 2014)

13. NIST Atomic Spectra Database, http://www.nist.gov/ $\mathrm{pml} /$ data/asd.cfm

14. D.-J. Shin, C.-W. Park, S.S. Kolesnikova, B. Khlevnoy, Final report on bilateral comparison APMP.PR-K1.a.12008 between KRISS (Korea) and VNIIOFI (Russia): Spectral irradiance from $250 \mathrm{~nm}$ to $2500 \mathrm{~nm}$, Metrologia 47, 02005 (2010)
15. ISO/IEC Guide 98:1995, Guide to the expression of uncertainty in measurement (International Organization for Standardization, Geneva, 1995)

16. S. Park, D.-H. Lee, Y.-W. Kim, S.-N. Park, Uncertainty evaluation for the spectroradiometric measurement of the averaged light-emitting diode intensity, Appl. Opt. 46, $2851-2858$ (2007)

17. IEC 60904-9:2007, Photovoltaic devices - Part 9: Solar simulator performance requirements, Edition 2.0 (International Electrotechnical Commission, Geneva, 2007) 